\title{
Error bounds for the approximative solution of restricted planar location problems
}

\author{
Barbara Käfer \\ Stefan Nickel* \\ University of Kaiserslautern \\ University of Kaiserslautern
}

16th October 1997

\begin{abstract}
Facility location problems in the plane play an important role in mathematical programming. When looking for new locations in modeling real-world problems, we are often confronted with forbidden regions, that are not feasible for the placement of new locations. Furthermore these forbidden regions may have complicated shapes. It may be more useful or even necessary to use approximations of such forbidden regions when trying to solve location problems.

In this paper we develop error bounds for the approximative solution of restricted planar location problems using the so called sandwich algorithm. The number of approximation steps required to achieve a specified error bound is analyzed. As examples of these approximation schemes, we discuss round norms and polyhedral norms. Also computational tests are included.
\end{abstract}

Keywords: Location Theory, Forbidden Regions, Approximation, Global Optimization, Applications

\footnotetext{
${ }^{*}$ Partially supported by a grant of the Deutsche Forschungsgemeinschaft
} 


\section{Introduction}

In the field of location theory, planar location problems have always played an important role. A large body of literature (see [DD84] and [Dre95]) is witness to the history of development of location theory and its various successful applications.

Since planar location problems have to deal with a geometrical model of real world problems the consideration of restrictions caused by nature reserves or unfavorable geographic conditions such as for example lakes is essential. As an extension to the basic model we introduce forbidden regions, in which the placement of new facilities is not allowed (see [HN94], [AM94], [HN95] and [Nic95]). These problems are called restricted location problems.

As one can easily imagine, forbidden regions may have very complicated shapes. Therefore it is sometimes impracticable or even not possible to solve a restricted location problem with the original forbidden region. Instead we will develop algorithms using a polyhedral approximation of the forbidden region, with which we can easily solve the restricted location problem, while guaranteeing a given error bound. For this aim we construct inner and outer polygonal approximations of the forbidden region. We then know that the solution of the original problem is bounded by the solutions we get by solving the problem on the approximating polygons.

More formally:

We are given a set of existing facilities $E x=\left\{E x_{1}, \ldots, E x_{M}\right\}$ represented by points in the plane with coordinates $E x_{m}=\left(a_{m}, b_{m}\right)$ for $m \in \mathcal{M}:=\{1, \ldots, M\}$. Every existing facility is assigned a positive weight $w_{m}$ for $m \in \mathcal{M}$. In some extensions we will discuss negative weights, too. We look for a new facility $X$ that fulfills $X \notin i n t(\mathcal{R})$, where $\mathcal{R} \subseteq \mathbb{R}^{2}$ is a connected set, that represents a forbidden region. To measure the quality of the solution we use the following criterion

$$
\sum_{m \in \mathcal{M}} w_{m} d\left(E x_{m}, X\right)=: f(X)
$$

where $d(R, S)$ means the distance between two points $R$ and $S$ in the plane, measured by some metric $d$.

The corresponding optimization problem with the objective function $f(X)$ is

$$
\min _{X \in \mathcal{F}} f(X)
$$

which is called in the literature Weber- or Median- or Minisum-Problem.

The set $\mathcal{F}$ over which we minimize is called the feasible region, defined by $\mathcal{F}:=\mathbb{R}^{2} \backslash \operatorname{int}(\mathcal{R})$. Since the naming conventions in location theory are not unique, to avoid ambiguity and to get an overview over the manifold area of location problems we want to use a classification scheme developed for location problems in [Ham95], [HN96] and [HNS96].

We have the following five position classification

$$
\operatorname{pos} 1 / \operatorname{pos} 2 / \operatorname{pos} 3 / \operatorname{pos} 4 / \operatorname{pos} 5
$$


where the meaning of each position is explained in the following table:

\begin{tabular}{|c|l|l|}
\hline Position & Meaning & Usage (Examples) \\
\hline \hline 1 & number of new facilities & \\
\hline \multirow{3}{*}{2} & & $\mathbf{P}$ planar location problem \\
& type of problem & $\mathbf{D}$ discrete location problem \\
& & $\mathbf{G}$ location problem on a network \\
\hline 3 & special assumptions and restrictions & $w_{m}=1$ all weights are equal \\
\hline \multirow{2}{*}{4} & type of distance function & $l_{1}$ Manhattan metric \\
& & $\gamma$ a general gauge \\
\hline \multirow{2}{*}{5} & type of objective function & $\sum$ Median problem \\
& & max Center problem \\
\hline
\end{tabular}

If we do not make any special assumptions in a position, we indicate this by a $\bullet$. For example, a $\bullet$ in position 4 means that we are talking about any distance function. In the classical case, where $\mathcal{F}=\mathbb{R}^{2}$ we denote the set of optimal solutions of $1 / P / \bullet / \bullet / \sum$ by $\mathcal{X}^{*}$.

The set of optimal locations of $1 / P / \mathcal{R} / \bullet / \sum$ is denoted $\mathcal{X}_{\mathcal{R}}^{*}$.

The rest of the paper is organized as follows. In the second section we state some basic properties for restricted location problems and define the class of distance functions we will investigate in this paper. Further we will discuss some principal solution techniques and introduce the sandwich algorithm. The third section is concerned with the deduction of the error bounds for approximating solutions of restricted location problems.

It should be noted, that although we restrict ourselves in this paper to the case of one forbidden region, all algorithms can be modified to handle also the case of multiple forbidden regions (see [HN95] and [Nic95]). 


\section{Basic principles and tools}

\subsection{Solution of restricted location problems}

We assume in the following that $\mathcal{X}^{*} \subseteq \operatorname{int}(\mathcal{R})$, to avoid trivial solutions. The weights $w_{m}$ are without loss of generality always assumed to be normalized. This means $\sum_{m \in \mathcal{M}} w_{m}=1$ in the case of all weights being positive. If negative weights are involved we assume for $w^{+}:=\sum_{w_{m} \geq 0} w_{m}$ and $w^{-}:=-\sum_{w_{m} \leq 0} w_{m}$, that $\max \left\{w^{+}, w^{-}\right\}=1$. By default all weights $w_{m}$ are positive and extensions to negative weights are mentioned explicitly. After these technical remarks we can state a fundamental property of restricted location problems:

Theorem 2.1 (see [HN95]) Let $h$ be a convex objective function for $1 / P / \mathcal{R} / \bullet / \bullet$ and $\partial \mathcal{R}$ the boundary of $\mathcal{R}$. If $\mathcal{X}^{*} \subseteq$ int $(\mathcal{R})$, then

$$
\mathcal{X}_{\mathcal{R}}^{*} \subseteq \partial \mathcal{R}
$$

If we allow negative weights $w_{m}$, the objective function need not to be convex anymore. Therefore we have to deal with several local minima and the fact that an optimal location for $1 / P / \mathcal{R} / \bullet / \sum$ can be either a local optimum outside of $\mathcal{R}$ or on the boundary of $\mathcal{R}$ (for details see [ND96]). In this case the approximation tool we develop in the following does not only serve to locate a best location on $\partial \mathcal{R}$. It also helps to decide if a search on $\partial \mathcal{R}$ is necessary or if we are done with the smallest of the local minima outside of $\mathcal{R}$ and can forget about the boundary of the forbidden region.

In general we assume non-negative weights to make the paper easier to read. However, we describe at the appropriate places in the text how the extension to the case with negative weights can be done.

To find an optimal solution of the considered restricted location problems we have to find the maximal level curve contained in the set $\mathcal{R}$, where we will denote by level curve the set

$$
L_{=}(z, h):=\left\{X \in \mathbb{R}^{2}: h(X)=z\right\} .
$$

The real number $z$ is called level of $L_{=}(z, h)$. For the sake of simplicity, we denote by $L_{=}(z)$ the level curve $L_{=}(z, f)$. For a more detailed discussion of restricted location problems see [HN95] and [Ham95].

The complexity of the determination of the optimal level curve heavily depends on the structure of the forbidden region $\mathcal{R}$, which leads to the consideration of an approximation approach, as explained in the introduction. We construct inner and outer approximations $\bar{P}$ and $\underline{P}$, and solve the problems $1 / P / \mathcal{R}=\bar{P} / \bullet / \sum$ and $1 / P / \mathcal{R}=\underline{P} / \bullet / \sum$, respectively. That is, we calculate two level curves enclosing the optimal level curve for the original forbidden region. The difference of the objective values of an outer and an inner approximation gives us an error bound for the optimal objective value.

Since these approximations are calculated with respect to the objective value of the optimal solution and the approximative solutions, the shape and location of the level curves have to be analyzed in detail. 
In the considered objective function $f(X)$ with

$$
f(X)=\sum_{m \in \mathcal{M}} w_{m} d_{m}\left(X, E x_{m}\right) \quad \text { and } \quad \sum_{m \in \mathcal{M}} w_{m}=1
$$

the profit of a new facility for any existing facility is measured by a concept of distance $d(R, S)$ between points $R$ and $S$ in the plane. There are different possibilities of distance functions, that have different effects on the shape of the level curves and the solution algorithm.

In this paper we want to discuss first a problem with circles as level curves $\left(l_{2}^{2}\right)$ to give the reader some insight in the proving techniques needed for establishing error bounds. After that, results for a subclass of the polyhedral norms as distance functions are presented. This is interesting since the set of polyhedral norms is dense in the set of all norms (see [Mic87], [WW85] and [Nic95]). We give the definitions of these two types of distance functions in the remaining parts of this section, which are concerned with specific location problems. The last part of this section contains an introduction to the sandwich algorithm.

\subsection{The location problem $1 / P / \mathcal{R} / l_{2}^{2} / \Sigma$}

The $l_{2}^{2}$ - function is defined by

$$
l_{2}^{2}(X):=x_{1}^{2}+x_{2}^{2}
$$

with $X=\left(x_{1}, x_{2}\right)$.

In Section 2.1 we have seen that the boundary of $\mathcal{R}$ for any location problem with convex objective function contains $\mathcal{X}_{R}^{*}$. An interesting particularity for the $l_{2}^{2}$-distance function is, that the convexity of the objective function is guaranteed even for some negative weights as long as the optimal solution is finite. (see [DW91]).

In [FLMW92] it is shown that the level curves are circles. If the boundary of the forbidden region $\mathcal{R}$ is polygonal, we know how to calculate the maximal circle contained in $\mathcal{R}$. The next result (see [HN95]), implies an approximation procedure as explained in the introduction for forbidden regions with complicated shapes, where we do not know how to calculate the maximal contained circle.

Lemma 2.1 (see [HN95]) Let $\mathcal{R}=\left\{X \in \mathbb{R}^{2}: a_{i} X \leq b_{i}, a_{i} \in \mathbb{R}^{2}, b_{i} \in \mathbb{R}, i=1, \ldots, I\right\}$ be a convex polytope. For $i=1, \ldots, I$ let $P_{i}:=\left(p_{i_{1}}, p_{i_{2}}\right)$ be the orthogonal projection point of $\mathcal{X}^{*}$ on $\left\{X: a_{i} X=b_{i}\right\}$.

Then

$$
\mathcal{X}_{R}^{*}:=\operatorname{argmin}\left\{f\left(P_{i}\right): i=1 \ldots, I\right\}
$$

is the set of optimal solutions. 


\subsection{The location problem $1 / P / \mathcal{R} / \gamma_{B} / \Sigma$}

Definition 2.1 Let $B$ be a compact convex subset of $\mathbb{R}^{2}$, with $0 \in \operatorname{int}(B)$. The function $\gamma: \mathbb{R}^{2} \rightarrow \mathbb{R}_{0}^{+}$defined by

$$
\gamma(X):=\inf \{\lambda>0: X \in \lambda B\} \text { with } X \in \mathbb{R}^{2}
$$

is called the gauge of $X$ with respect to $B$.

As one can easily see, a gauge becomes a norm if $B$ is symmetric (see [DM85]). In the following we will consider a special type of symmetric gauges that are called block norms. The unit ball of a block norm is a convex polytope with extreme points $e_{1}, \ldots, e_{G}$, where $G$ is even and $e_{i}=e_{i+\frac{G}{2}}$, for $i=1, \ldots \frac{G}{2}$. . Therefore we can write

$$
\gamma_{B}(X)=\min \left\{\sum_{g=1}^{G} \lambda_{g}: X=\sum_{g=1}^{G} \lambda_{g} e_{g}, \lambda_{g} \geq 0 \text { for } g=1 \ldots G\right\}
$$

Let $d_{1}, \ldots, d_{G}$ be the halflines defined by the endpoints 0 and the points $e_{1}, \ldots, e_{G}$. We call $d_{1}, \ldots, d_{G}$ fundamental directions.

To be sure that we consider extreme points $e_{1}, \ldots, e_{G}$, where no point $e_{i}, i=1, \ldots, G$ is contained in the line connecting adjacent extreme points we define non-degenerated unit balls as follows.

Definition 2.2 A unit ball $B=\operatorname{conv}\left\{e_{1}, \ldots, e_{G}\right\}$ of a block norm with $G$ fundamental directions is called non-degenerated if for all fundamental directions $d_{g}$ with $g=1, \ldots, G$

$$
e_{g} \notin B^{\prime}:=\operatorname{conv}\left(\left\{e_{1}, \ldots, e_{G}\right\} \backslash\left\{e_{g}\right\}\right)
$$

holds.

Let the cone generated by two consecutive fundamental directions $d_{g}, d_{g+1}$ be denoted $\Gamma_{g}$, where we set $d_{G+1}:=d_{1}$. If the origin of the cone $\Gamma_{g}$ is shifted to a point $X \in \mathbb{R}^{2}$ we denote this cone $\Gamma_{g}(X)$.

We call $H_{g}^{-}$and $H_{g}^{+}$the halfspaces, bounded by the hyperplane generated by an halfline $d_{g}$, $g=1, \ldots, G$. In the following we write $H_{g}$ for a fixed one chosen from the two halfspaces $H_{g}^{-}$or $H_{g}^{+}$. The halfspace of an hyperplane in an existing facility $E x_{m}$ is denoted $H_{g}^{m}$.

For every existing facility we get $\frac{G}{2}$ lines, called construction lines and $\frac{G}{2}$ halfspaces. This is a consequence of the symmetry of the block norms. Since we consider for all existing facilities the same norm, we can assume the halfspaces to be ordered in the way that we have for all $g=1, \ldots, G / 2: H_{g}^{1} \subseteq H_{g}^{2} \subseteq \ldots \subseteq H_{g}^{M}$.

We add $H_{g}^{M+1}:=\mathbb{R}^{2}$ as the halfspace containing all halfspaces $H_{g}^{m}$ and get a decomposition of $\mathbb{R}^{2}$ into cells $<s_{1}, \ldots, s_{G / 2}>$ with $s_{i} \in\{1, \ldots, M+1\}$, for all $i=1, \ldots, \frac{G}{2}$ defined as

$$
\left\langle s_{1}, \ldots, s_{G / 2}\right\rangle:=\left\{\left(x_{1}, x_{2}\right):\left(x_{1}, x_{2}\right) \in \bigcap_{i=1}^{G / 2}\left(H_{i}^{s_{i}} \backslash H_{i}^{s_{i-1}}\right)\right\},
$$


where $H_{i}^{0}:=\emptyset \forall i$. With $\left\langle\mathbb{R}^{2}\right\rangle$ we denote the set of all cells. Note that this definition is a special case of the definition of elementary convex sets for general gauges by [DM85].

The objective function is linear in each cell $\left\langle s_{1}, \ldots, s_{G / 2}\right\rangle \in\left\langle\mathbb{R}^{2}\right\rangle$ (see, for example [Nic95]), which also holds for negative weights.

Moreover, for non-degenerated unit balls we get from the definition of polyhedral gauges.

Lemma 2.2 Let $\gamma_{B}$ be the polyhedral gauge of a non-degenerated unit ball $B=$ conv $\left\{e_{1}, \ldots, e_{G}\right\}$. Let $\Gamma_{\nu}$ be the cone generated by $d_{\nu}$ and $d_{\nu+1}$ where $d_{G+1}=d_{1}$ and $e_{G+1}=e_{1}$. Let $X \in \Gamma_{\nu}$. There are uniquely defined $\lambda_{1}$ and $\lambda_{2} \in \mathbb{R}_{+}$with:

$$
X=\lambda_{1} e_{\nu}+\lambda_{2} e_{\nu+1} \text { and } \gamma(X)=\lambda_{1}+\lambda_{2}
$$

Using Lemma 2.2 and the fact that for every $X \in \mathbb{R}^{2}$ there exists a cell $\left\langle s_{1}, \ldots, s_{G / 2}\right\rangle \in$ $\left\langle\mathbb{R}^{2}\right\rangle$ with $\left.X \in<s_{1}, \ldots, s_{G / 2}\right\rangle$, we can write

$$
f(X)=V_{1}\left(\left\langle s_{1}, \ldots, s_{G / 2}\right\rangle\right) \cdot x_{1}+V_{2}\left(\left\langle s_{1}, \ldots, s_{G / 2}\right\rangle\right) \cdot x_{2}+V\left(\left\langle s_{1}, \ldots, s_{G / 2}\right\rangle\right)
$$

with

$$
\begin{aligned}
& V_{1}\left(\left\langle s_{1}, \ldots, s_{G / 2}\right\rangle\right)=\sum_{g=1}^{G} \sum_{\substack{m \in \mathcal{M} w i t h \\
X-E x_{m} \in \Gamma_{g}}} w_{m} \cdot \frac{\left(e_{g}^{2}-e_{g+1}^{2}\right)}{e_{g}^{2} e_{g+1}^{1}-e_{g}^{1} e_{g+1}^{2}}, \\
& V_{2}\left(\left\langle s_{1}, \ldots, s_{G / 2}\right\rangle\right)=\sum_{g=1}^{G} \sum_{\substack{m \in \mathcal{M} \text { with } \\
X-E x_{m} \in \Gamma_{g}}} w_{m} \cdot \frac{\left(e_{g+1}^{1}-e_{g}^{1}\right)}{e_{g}^{2} e_{g+1}^{1}-e_{g}^{1} e_{g+1}^{2}},
\end{aligned}
$$

where $G+1=1$ as above and $V\left(\left\langle s_{1}, \ldots, s_{G / 2}\right\rangle\right)$ is constant for a fixed cell $\left\langle s_{1}, \ldots, s_{G / 2}\right\rangle \in$ $\left\langle\mathbb{R}^{2}\right\rangle$.

For axial symmetric block norms we have $\frac{\left(e_{g}^{2}-e_{g+1}^{2}\right)}{e_{g}^{2} e_{g+1}^{1}-e_{g}^{1} e_{g+1}^{2}} \leq 1$ and $\frac{\left(e_{g+1}^{1}-e_{g}^{1}\right)}{e_{g}^{2} e_{g+1}^{1}-e_{g}^{1} e_{g+1}^{2}} \leq 1$ (see [K $\left.\ddot{9} 6\right]$ ). By scaling the weights as explained at the beginning of this section the same is true for negative weights, too.

In the general polyhedral gauge case we denote by $m_{1}$ the maximum of the terms $\frac{\left(e_{g}^{2}-e_{g+1}^{2}\right)}{e_{g}^{2} e_{g+1}^{1}-e_{g}^{1} e_{g+1}^{2}}$ and by $m_{2}$ the maximum of the terms $\frac{\left(e_{g+1}^{1}-e_{g}^{1}\right)}{e_{g}^{2} e_{g+1}^{1}-e_{g}^{1} e_{g+1}^{2}}$ and get $V_{1}$ and $V_{2}$ bounded by $M \cdot m_{1}$ and $M \cdot m_{2}$. This is also a bound for the case with negative weights.

Remark: We know that the objective function is piece-wise linear and therefore the level curves have be polygons. The linear pieces of the level curves can change their slopes only at the intersection points with the construction lines. We then know that an optimum $X_{\mathcal{R}}^{*} \in \mathcal{X}_{\mathcal{R}}^{*}$ needs to be on a construction line and by Theorem $2.1 \mathcal{X}_{\mathcal{R}}^{*} \subseteq \partial \mathcal{R}$. The resulting solution algorithm is then straightforward and consists of intersecting $\partial \mathcal{R}$ with the construction lines and choosing the best of the intersection points ( for details see $[\mathrm{Nic} 95])$. 
Therefore we obviously do not always need the approximation approach explained in the introduction for solving just one single location problem. A solution algorithm simply consists of calculating the intersection points and choosing the one with the smallest objective function value.

There are two reasons, why we consider it important to analyze the behavior of location problems with block norms and solving them with the approximation algorithm.

Once an approximation is established, we are able to analyze the behavior of different types of objective functions and give approximate estimates, without explicitly solving the location problem.

The second application is even more important. Round norms are often hard to handle, while for block norms algorithms are available. It is also known, that polyhedral norms are dense in the set of all norms (see [Val64] and [WW85]). Therefore it is interesting to approximate the unit ball of a round norm by the one of a polyhedral norm and estimate the resulting error in the new objective function.

\subsection{The Sandwich Algorithm}

For convex sets there exists an efficient approximation algorithm which is called the sandwich algorithm (see [Rot92]). We will use an extension of the original sandwich algorithm, developed for convex functions in [BHR91] to convex sets (see [Rot92]).

The original sandwich algorithm provides piecewise linear approximations for a convex function $f$ that is bounded on an interval $[a, b]$ and that has finite right and left derivatives $f^{+}(a)$ and $f^{-}(b)$ at the endpoints $a$ and $b$ of the interval.

For a finite partition $a=t_{0}<t_{1}<\ldots<t_{n}=b$ of the interval $[a, b]$ we denote by $f_{i}^{+}$and $f_{i}^{-}$the right and left derivatives for a point $t_{i}$.

We construct an upper approximation $u(t)$ and a lower approximation $l(t)$ for the function $f$ where

$$
\begin{aligned}
& u(t)=f\left(t_{i}\right)+\frac{f\left(t_{i+1}\right)-f\left(t_{i}\right)}{t_{i+1}-t_{i}}\left(t-t_{i}\right) \\
& l(t)=\max \left\{f\left(t_{i}\right)+f_{i}^{+}\left(t-t_{i}\right), f\left(t_{i+1}\right)+f_{i+1}^{-}\left(t-t_{i+1}\right)\right\}
\end{aligned}
$$

and $t \in\left[t_{i}, t_{i+1}\right]$.

The error $\varepsilon$ for this approximation is defined as $\max _{0 \leq i \leq n-1}\left\{u\left(t_{i}^{*}\right)-l\left(t_{i}^{*}\right)\right\}$, where in an interval $\left[t_{i}, t_{i+1}\right]$ the point $t_{i}^{*}$, where the maximal error is attained is defined by

$$
t_{i}^{*}=\frac{f\left(t_{i+1}\right)-f\left(t_{i}\right)+f_{i}^{+} t_{i}-f_{i+1}^{-} t_{i+1}}{f_{i}^{+}-f_{i+1}^{-}} .
$$

This error therefore is the maximal vertical error. Starting with the interval $[a, b]$, we construct $u(t)$ and $l(t)$ for $t_{i}=a$ and $t_{i+1}=b$. If the maximal error in $[a, b]$ or any following interval $\left[t_{i}, t_{i+1}\right]$ exceeds the given tolerance we divide the interval according to a dissection rule. 
By continuing like that we get approximations $u_{n}, l_{n}$ with:

$$
l_{n} \leq f \leq u_{n} \quad \text { for } n=0,1, \ldots
$$

See [Rot92] for further details, the formulation of the algorithm and possible dissection rules.

The dissection rule that is of most interest also for convex sets is the chord rule. Considering the part of the boundary between two existing dissection points, following this rule, we choose the point whose slope is equal to the slope of the line connecting the two existing points.

In the following picture the way the chord rule proceeds is illustrated.

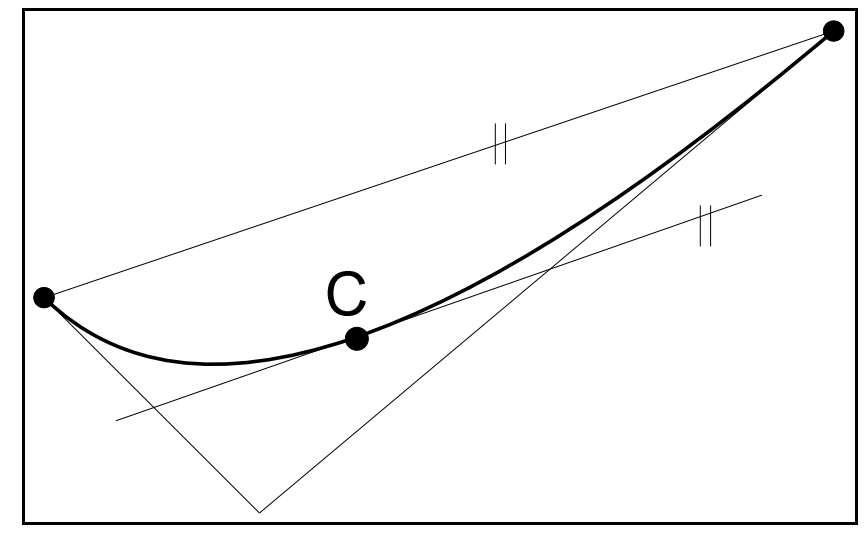

Figure 1: chord rule

We use this rule because it is the only rule that is insensitive to transformations of the coordinate system. For convex sets we need this property to ensure that the dissection points are the same even if we rotate the set.

In the following we apply this rule and refer to [Rot92] for some other dissection rules and the corresponding error bounds.

We do not discuss the error bounds developed for convex functions in detail (see [Rot92]). They easy carry over to convex sets $\mathcal{R}$ by dividing the boundary $\partial \mathcal{R}$ of $\mathcal{R}$ in four pieces and by applying the approximation on every piece. This four pieces are bounded from the tangent points of the smallest rectangle parallel to the $\mathrm{x}$ - and $\mathrm{y}$-axis containing $\mathcal{R}$. If we approximate convex sets we have no canonical way of measuring the error like it is the case with convex functions. Therefore we use the Hausdorff distance, which is defined for an inner approximation $\bar{P}$ and an outer approximation $\underline{P}$ as:

$$
\delta^{H}(\underline{P}, \bar{P})=\sup _{X \in \underline{P}} \inf _{Y \in \bar{P}} l_{2}(X, Y)
$$

where $l_{2}(X, Y)$ denotes the Euclidean distance between $X$ and $Y$. The normal distance between two sets is always meant in the following as the minimal distance between any two points of these sets. 
Using that the Hausdorff distance is bounded by the maximal vertical distance, in [Rot92] the following result is proved:

Theorem 2.2 Using the chord rule, to approximate a convex planar figure $P$ of circumference $D$ by two n-gones with an error of at most $\varepsilon$ we need at most

$$
\tilde{m}(D, \epsilon):=\max \left\{4,\left\lceil\sqrt{\frac{8 D}{\varepsilon}}+2\right\rceil\right\} .
$$

additional dissection points. 


\section{Error bounds}

Approximating the forbidden region by an outer and an inner polyhedron provides an approximate solution of the original problem and its objective function value and allows us to give upper and lower bounds for the objective value of the exact optimal solution. The set of optimal points on the outer approximation $1 / P / \underline{P} / \bullet / \sum$ is denoted by $\mathcal{X}_{\underline{P}}^{*}$, the set of optimal points on the inner approximation $1 / P / \bar{P} / \bullet / \sum$ is denoted by $\mathcal{X}_{\bar{P}}^{*}$. We will use the error bounds developed in the last section to get error bounds for the objective value of location problems. The difference between the optimal objective values $f\left(\mathcal{X}_{\underline{P}}^{*}\right)$ of the outer and $f\left(\mathcal{X}_{\bar{P}}^{*}\right)$ of the inner approximation is denoted by $\varepsilon$. Assuming a specified approximation procedure we are able to state error bounds in advance. In the following we will consider the chord rule. Further we denote by $\underline{P}_{0}$ the smallest rectangle parallel to the $x$ - and $y$ - axis containing $\mathcal{R}$. According to Section 2.4 this is always the first outer approximation and all other outer approximations $\underline{P}$ are contained in $\underline{P}_{0}$.

\subsection{Error bounds for $1 / P / \mathcal{R} / l_{2}^{2} / \Sigma$}

In Section 2.2 the structure of the level curves for $1 / P / \mathcal{R} / l_{2}^{2} / \sum$ was described. Further we introduced the necessary steps to find the solution. We will now give some more details on how the approximation approach works and some more additional properties. The objective function of $1 / P / \mathcal{R} / l_{2}^{2} / \sum$ possesses the following property.

Lemma 3.1 Let $f$ be the objective function of $1 / P / \mathcal{R} / l_{2}^{2} / \sum$ and $P$ and $\hat{P}$ two points in $\mathbb{R}^{2}$. Then

$$
f(P)-f(\hat{P})=l_{2}^{2}\left(P, \mathcal{X}^{*}\right)-l_{2}^{2}\left(\hat{P}, \mathcal{X}^{*}\right)
$$

\section{Proof:}

$$
\begin{aligned}
& f(P)-f(\hat{P}) \\
= & \sum_{m \in \mathcal{M}} w_{m} l_{2}^{2}\left(P, E x_{m}\right)-\sum_{m \in \mathcal{M}} w_{m} l_{2}^{2}\left(\hat{P}, E x_{m}\right) \\
= & \sum_{m \in \mathcal{M}} w_{m}\left(\left(p_{1}^{2}-2 p_{1} a_{m}+a_{m}^{2}+p_{2}^{2}-2 p_{2} b_{m}+b_{m}^{2}\right)-\right. \\
& \left.\left({\hat{p_{1}}}^{2}-2 \hat{p_{1}} a_{m}+a_{m}^{2}+\hat{p}_{2}{ }^{2}-2 \hat{p_{2}} b_{m}-b_{m}^{2}\right)\right) \\
= & \left(p_{1}-\sum_{m \in \mathcal{M}} w_{m} a_{m}\right)^{2}+\left(p_{2}-\sum_{m \in \mathcal{M}} w_{m} b_{m}\right)^{2}-\left(\left(\hat{p_{1}}-\sum_{m \in \mathcal{M}} w_{m} a_{m}\right)^{2}+\left(\hat{p_{2}}-\sum_{m \in \mathcal{M}} w_{m} b_{m}\right)^{2}\right) \\
= & l_{2}^{2}\left(P, \mathcal{X}^{*}\right)-l_{2}^{2}\left(\hat{P}, \mathcal{X}^{*}\right)
\end{aligned}
$$

The last equation holds, since $\mathcal{X}^{*}=\left(\sum_{m \in \mathcal{M}} w_{m} a_{m}, \sum_{m \in \mathcal{M}} w_{m} b_{m}\right)$ is the unique optimal solution of $1 / P / \bullet / l_{2}^{2} / \sum$. 
Lemma 3.2 Let $\mathcal{R}$ be a convex set with $\mathcal{X}^{*} \in \operatorname{int}(\mathcal{R}), \bar{P}$ and $\underline{P}$ inner and outer approximations of $\mathcal{R}$ respectively. Further let $\left[T_{h}, T_{h+1}\right]$ be an edge of the inner polygon $\bar{P}$, containing $X_{\underline{P}}^{*} \in \mathcal{X}_{\underline{P}}^{*}$. For the outer polygon $\underline{P}$ let $X_{\bar{P}}^{*} \in \mathcal{X}_{\bar{P}}^{*}$ on the edge $\left[S_{k}, S_{k+1}\right]$. Then the following holds:

$$
l_{2}\left(X_{\underline{P}}^{*}, \mathcal{X}^{*}\right)-l_{2}\left(X_{\bar{P}}^{*}, \mathcal{X}^{*}\right)<\delta^{H}(\bar{P}, \underline{P}) .
$$

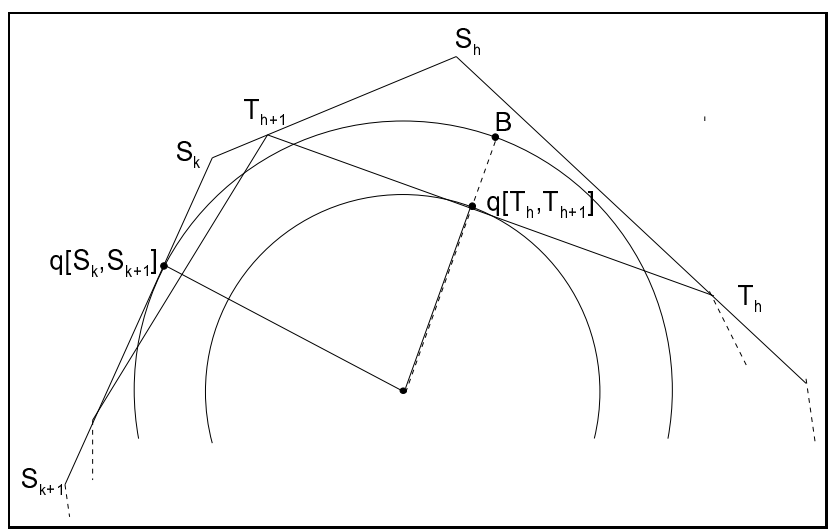

Figure 2: Illustration to the proof of Lemma 3.2

Proof: First note that by Lemma $2.1 X_{P}^{*}$ is the projection of $\mathcal{X}^{*}$ onto $\left[S_{k}, S_{k+1}\right]$, denoted by $q\left[S_{k}, S_{k+1}\right]$ and $X_{P}^{*}$ is the projection of $\mathcal{X}^{*}$ onto $\left[T_{h}, T_{h+1}\right]$, denoted by $q\left[T_{h}, T_{h+1}\right]$. Let $K\left(\mathcal{X}^{*}, d\left(\mathcal{X}^{*}, X_{\underline{P}}^{*}\right)\right)$ be the circle with center $\mathcal{X}^{*}$ and radius $\left.l_{2}\left(\mathcal{X}^{*}, X_{\underline{P}}^{*}\right)\right)$ and let $B$ be the point in the triangle $T_{h}, T_{h+1}, S_{h}$ that fulfills:

$$
B \in K\left(\mathcal{X}^{*}, d\left(\mathcal{X}^{*}, X_{\bar{P}}^{*}\right)\right) \text { and }\left[B, X_{\underline{P}}^{*}\right] \perp\left[T_{h}, T_{h+1}\right] .
$$

Furthermore let $S_{l}$ be the edge where the Hausdorff distance is attained. Then:

$$
\begin{aligned}
l_{2}\left(X_{\underline{P}}^{*}, \mathcal{X}^{*}\right)-l_{2}\left(X_{\bar{P}}^{*}, \mathcal{X}^{*}\right) & =l_{2}\left(B, \mathcal{X}^{*}\right)-l_{2}\left(X_{\bar{P}}^{*}, \mathcal{X}^{*}\right) \\
& =l_{2}\left(B, X_{\bar{P}}^{*}\right) \\
& =l_{2}\left(B,\left[T_{h}, T_{h+1}\right]\right) \\
& <l_{2}\left(S_{h},\left[T_{h}, T_{h+1}\right]\right. \\
& \leq l_{2}\left(S_{l},\left[T_{l}, T_{l+1}\right]\right) \\
& =\delta^{H}(\bar{P}, \underline{P}) .
\end{aligned}
$$

Lemma 3.3 The assumptions are the same as in Lemma 3.2. Further denote by $D$ the circumference of $\mathcal{R}$. Then the following holds:

- $l_{2}\left(X_{\underline{P}}^{*}, \mathcal{X}^{*}\right) \leq \frac{1}{4} D$. 
- $l_{2}\left(X_{\bar{P}}^{*}, \mathcal{X}^{*}\right) \leq \frac{D}{2 \pi}$.

Proof: Let $l$ be the length of the longest side of $\underline{P}_{0}$. Since the level curves are circles we have

$$
l_{2}\left(X_{\underline{P}}^{*}, \mathcal{X}^{*}\right) \leq \frac{1}{2} l
$$

By the definition of $\underline{P}_{0}$ we have further that $l \leq \frac{1}{2} D$. So we get in total

$$
l_{2}\left(X_{\underline{P}}^{*}, \mathcal{X}^{*}\right) \leq \frac{1}{4} D .
$$

By $D_{L}$ we denote the circumference of the level curve passing through $X_{\bar{P}}^{*}$. Then we get

$$
D_{L}=2 \pi l_{2}\left(X_{P}^{*}, \mathcal{X}^{*}\right) \leq D
$$

resulting in

$$
l_{2}\left(X_{\bar{P}}^{*}, \mathcal{X}^{*}\right) \leq \frac{D}{2 \pi}
$$

We are now able to relate the approximative solutions to the Hausdorff distance of $\bar{P}$ and $\underline{P}$.

Lemma 3.4 The assumptions are again the same as in Lemma 3.2. Let $D$ be the circumference of $\mathcal{R}$ and let $f$ be the objective function of an $1 / P / \mathcal{R} / l_{2}^{2} / \sum$ location problem. Then:

where $\rho:=\frac{1}{4}+\frac{1}{2 \pi}$.

$$
f\left(X_{\underline{P}}^{*}\right)-f\left(X_{\bar{P}}^{*}\right)<\delta^{H}(\underline{P}, \bar{P}) \rho D
$$

Proof: Using Lemma 3.2 and Lemma 3.3 we get:

$$
\begin{aligned}
f\left(X_{\underline{P}}^{*}\right)-f\left(X_{\bar{P}}^{*}\right) & =l_{2}^{2}\left(X_{\underline{P}}^{*}, \mathcal{X}^{*}\right)-l_{2}^{2}\left(X_{\bar{P}}^{*}, \mathcal{X}^{*}\right) \\
& =l_{2}\left(X_{\underline{P}}^{*}, \mathcal{X}^{*}\right)^{2}-l_{2}\left(X_{\bar{P}}^{*}, \mathcal{X}^{*}\right)^{2} \\
& =\left(l_{2}\left(X_{\underline{P}}^{*}, \mathcal{X}^{*}\right)-l_{2}\left(X_{\bar{P}}^{*}, \mathcal{X}^{*}\right)\right) \cdot\left(l_{2}\left(X_{\underline{P}}^{*}, \mathcal{X}^{*}\right)+l_{2}\left(X_{\bar{P}}^{*} \mathcal{X}^{*}\right)\right) \\
& <\delta^{H}(\underline{P}, \bar{P}) \cdot\left(\frac{1}{4} D+\frac{D}{2 \pi}\right)
\end{aligned}
$$

Using these lemmas we are able to apply the sandwich algorithm error bounds to $1 / P / \mathcal{R} / l_{2}^{2} / \sum$.

Theorem 3.1 Let $\mathcal{R}$ be a convex restriction set of circumference $D$ in $\mathbb{R}^{2}$. To approximate the optimal objective value of $1 / P / \mathcal{R} / l_{2}^{2} / \sum$ with an maximal error of at most $\varepsilon$, we need (assuming the choice of the chord rule) at most

$$
m^{l_{2}^{2}}(D, \varepsilon):=\max \left\{4,\left\lceil D \sqrt{\frac{8 \rho}{\varepsilon}}+2\right\rceil\right\}
$$

dissection points. 
Proof: Theorem 2.2 tells us, that after

$$
\tilde{m}(D, \varepsilon)=\max \{4,\lceil\sqrt{8 D / \varepsilon}+2\rceil\}
$$

iterations $\delta^{H}(\bar{P}, \underline{P}) \leq \varepsilon$ holds.

From Lemma 3.2 and Lemma 3.4 it follows:

$$
\delta^{H}(\bar{P}, \underline{P}) \leq \frac{\varepsilon}{\rho D} \quad \Longrightarrow \quad f\left(X_{\underline{P}}^{*}\right)-f\left(X_{P}^{*}\right)<\varepsilon
$$

and therefore after

$$
\begin{aligned}
\tilde{m}\left(D, \frac{\varepsilon}{\rho D}\right) & =\max \left\{4,\left\lceil\sqrt{\left(\frac{8 D^{2} \rho}{\varepsilon}\right)}+2\right\rceil\right\} \\
& =\max \left\{4,\left\lceil D \sqrt{\frac{8 \rho}{\varepsilon}}+2\right\rceil\right\}=m^{l^{2}}(D, \varepsilon)
\end{aligned}
$$

iterations $f\left(X_{\underline{P}}^{*}\right)-f\left(X_{\bar{P}}^{*}\right)<\varepsilon$ holds.

\subsection{Error bounds for $1 / P / \mathcal{R} / \gamma_{B} / \Sigma$}

In Section 2 we have showed the linearity of the objective function in the cells generated by the construction lines of $1 / P / \mathcal{R} / \gamma_{B} / \sum$. We will use this linearity to derive error bounds. The bounds will be given for axial symmetric block norms $\gamma_{B}$ under no assumption for the weights (see Section 2.3 and remember, that the bounds for the linear factors of the objective functions are the same for positive or negative weights). We replace $\sqrt{2}$ by $\sqrt{\left(M \cdot m_{1}\right)^{2}+\left(M \cdot m_{2}\right)^{2}}$ (see Section 2.3) in the general polyhedral gauge case.

Lemma 3.5 Let $f$ be the objective function of $1 / P / \mathcal{R} / \gamma_{B} / \sum$, where $\gamma_{B}$ is a block norm that is axial symmetric.

a) For points $X, Y \in\left\langle s_{1}, \ldots, s_{G / 2}\right\rangle$ with $f(X)=z_{1}$ and $f(Y)=z_{2}$

$$
f(X)-f(Y) \leq \sqrt{2} \cdot d_{\left\langle s_{1}, \ldots, S_{G / 2}\right\rangle}\left(L_{z_{1}}, L_{z_{2}}\right)
$$

holds, where $d_{\left\langle s_{1}, \ldots, s_{G / 2}\right\rangle}\left(L_{z_{1}}, L_{z_{2}}\right)$ denotes the $l_{2}$-distance of the level curves $L_{z_{1}}$ through $X$ and $L_{z_{2}}$ through $Y$ in $\left\langle s_{1}, \ldots, s_{G / 2}\right\rangle$.

b) For points $X, Y \in \mathbb{R}^{2}$ with $f(X)=z_{1}$ and $f(Y)=z_{2}$

$$
f(X)-f(Y) \leq \sqrt{2} \cdot \min _{X \in L_{z_{1}}, Y \in L_{z_{2}}} l_{2}(X, Y)
$$

holds. 
Proof: a) In Section 2 we showed that $f$ can be written in a cell as follows:

$$
\begin{aligned}
& f(X)=z_{1}=V_{1}\left(s_{1}, \ldots, s_{G / 2}\right) x_{1}+V_{2}\left(s_{1}, \ldots, s_{G / 2}\right) x_{2}+V\left(s_{1}, \ldots, s_{G / 2}\right) \text { and } \\
& f(Y)=z_{2}=V_{1}\left(s_{1}, \ldots, s_{G / 2}\right) y_{1}+V_{2}\left(s_{1}, \ldots, s_{G / 2}\right) y_{2}+V\left(s_{1}, \ldots, s_{G / 2}\right) .
\end{aligned}
$$

Therefore:

$$
d_{\left\langle s_{1}, \ldots, s_{G / 2}\right\rangle}\left(L_{=}\left(z_{1}\right), L_{=}\left(z_{2}\right)\right)=\frac{z_{1}-z_{2}}{\sqrt{\underbrace{V_{1}\left(s_{1}, \ldots, s_{G / 2}\right)^{2}}_{\leq 1}+\underbrace{V_{2}\left(s_{1}, \ldots, s_{G / 2}\right)^{2}}_{\leq 1}}} .
$$

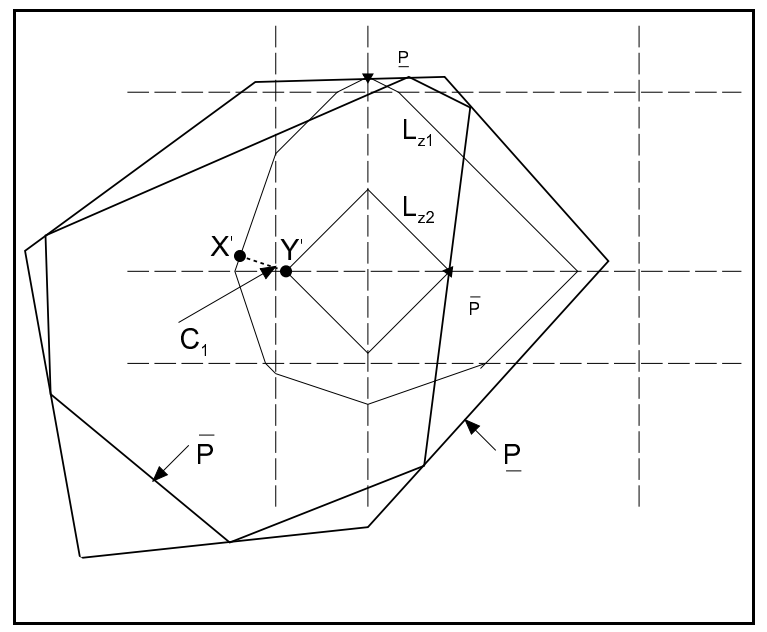

Figure 3: Illustration to the proof of Lemma 3.5 for the $l_{1}$-norm (four fundamental directions)

b) Let $X^{\prime} \in L_{z_{1}}, Y^{\prime} \in L_{z_{2}}$ with $l_{2}\left(X^{\prime}-Y^{\prime}\right)=\inf _{X \in L_{z_{1}}, Y \in L_{z_{2}}} l_{2}(X-Y)$. Further let $X^{\prime}$ be in $\left\langle s_{1}, \ldots, s_{G / 2}\right\rangle, Y^{\prime}$ be in $\left\langle s_{1}+t_{1}, \ldots, s_{G / 2}+t_{G / 2}\right\rangle$. The line connecting $X^{\prime}$ and $Y^{\prime}$ intersects $\sum_{i=1}^{G / 2}\left|t_{i}\right|$ construction lines. Let $C_{i}$ denote for $i=1, \ldots, p$ the intersection points ordered from $C_{1}:=X^{\prime}$ to $C_{p}:=Y^{\prime}$. Intersection points where several construction lines coincide are counted for every construction line. If there are $k$ construction lines to coincide at the same point, we have $k$ points $C_{l+1}, \ldots, C_{l+k}$ with $C_{l+1}=C_{l+2}=\ldots=C_{l+k}$.

From Lemma 3.5 a) we know:

$$
\begin{aligned}
f\left(X^{\prime}\right)-f\left(C_{1}\right)= & \sqrt{V_{1}\left(s_{1}, \ldots, s_{G / 2}\right)^{2}+V_{2}\left(s_{1}, \ldots, s_{G / 2}\right)^{2}} \cdot l_{2}\left(X^{\prime}, C_{1}\right), \\
f\left(C_{p}\right)-f\left(Y^{\prime}\right)= & \sqrt{V_{1}\left(s_{1}+t_{1}, \ldots, s_{G / 2}+t_{G / 2}\right)^{2}+V_{2}\left(s_{1}+t_{1}, \ldots, s_{G / 2}+t_{G / 2}\right)^{2}} \\
& \cdot l_{2}\left(C_{p}, Y^{\prime}\right), \\
f\left(C_{i}\right)-f\left(C_{i+1}\right)= & \sqrt{V_{1}\left(\operatorname{cell}\left(C_{i}, C_{i+1}\right)\right)^{2}+V_{2}\left(\operatorname{cell}\left(C_{i}, C_{i+1}\right)\right)^{2}} \cdot l_{2}\left(C_{i}, C_{i+1}\right), \\
\text { if } C_{i} \neq & C_{i+1} \text { and } \\
f\left(C_{i}\right)-f\left(C_{i+1}\right)= & 0 \\
\text { if } C_{i}= & C_{i+1}, \\
\forall i=1, \ldots, p, &
\end{aligned}
$$


where $\operatorname{cell}\left(C_{i}, C_{i+1}\right)$ denotes the cell which contains the whole line segment $\left[C_{i}, C_{i+1}\right]$. Summing up, we get

$$
\begin{array}{rll}
f(X)-f(Y) & =f\left(X^{\prime}\right)-f\left(Y^{\prime}\right) \\
\underset{\text { part a) }}{\leq} & \sqrt{2} \cdot\left(l_{2}\left(X^{\prime}, C_{1}\right)+l_{2}\left(C_{p+q}, Y^{\prime}\right)+\sum_{i=1}^{p-1} l_{2}\left(C_{i}, C_{i+1}\right)\right) \\
& =\sqrt{2} \cdot l_{2}\left(X^{\prime}, Y^{\prime}\right) \\
& =\sqrt{2} \cdot \min _{X \in L_{z_{1}}, Y \in L_{z_{2}}} l_{2}(X-Y) .
\end{array}
$$

Lemma 3.6 Let $f$ be the objective function of $1 / P / \mathcal{R} / \gamma_{B} / \sum$ with an axial symmetric unit circle $B$. Let $\bar{P}$ be an inner approximation of $\mathcal{R}$, let $\underline{P}$ be an outer approximation of $\mathcal{R}$. Additionally let $X_{P}^{*} \in \mathcal{X}_{\bar{P}}^{*}$ and $X_{\underline{P}}^{*} \in \mathcal{X}_{\underline{P}}^{*}$ with $f\left(X_{\bar{P}}^{*}\right)=z_{1}$ and $f\left(X_{\underline{P}}^{*}\right)=z_{2}$.

Then:

$$
f\left(X_{\underline{P}}^{*}\right)-f\left(X_{\bar{P}}^{*}\right) \leq \sqrt{2} \cdot \delta^{H}(\bar{P}, \underline{P}) .
$$

Proof: From Lemma 3.5 we know that

$$
\begin{aligned}
f\left(X_{\bar{P}}^{*}\right)-f\left(X_{\underline{P}}^{*}\right) & \leq \sqrt{2} \min _{X \in L_{z_{1}}} \min _{Y \in L_{z_{2}}} l_{2}(X, Y) \\
& \leq \sqrt{2} \min _{X \in L_{z_{1}}} l_{2}\left(X, X_{\underline{P}}^{*}\right) \\
& \leq \sqrt{2} \min _{X \in \underline{P}} l_{2}\left(X, X_{\underline{P}}^{*}\right) \\
& \leq \sqrt{2}\left(\sup _{X \in \underline{P}} \inf _{Y \in \bar{P}} l_{2}(X, Y)\right) \\
& =\sqrt{2} \delta^{H}(\bar{P}, \underline{P}) .
\end{aligned}
$$

From the preceding lemmas we have

$$
\delta^{H}(\bar{P}, \underline{P}) \leq \frac{\varepsilon}{\sqrt{2}} \Longrightarrow f\left(X_{\underline{P}}^{*}\right)-f\left(X_{\bar{P}}^{*}\right) \leq \varepsilon .
$$

and we get analogous to Theorem 3.1

Theorem 3.2 Let $\mathcal{R}$ be a forbidden region of circumference $D$ in $\mathbb{R}^{2}$, let $\gamma_{B}$ be an axial symmetric block norm. To approximate the objective value of $1 / P / \mathcal{R} / \gamma_{B} / \sum$ with an error of at most $\varepsilon$ we need at most

$$
m^{\gamma}(D, \varepsilon):=\max \left\{4,\left\lceil\sqrt{\frac{8 \sqrt{2} D}{\varepsilon}}+2\right\rceil\right\}
$$

additional dissection points. 


\section{Computational results}

For computational results of the general sandwich algorithm the reader is referred to [Rot92], where computational experiments for the different partition rules and for several convex functions have been made. Due to the fact, that we are interested in a priori error bounds, the construction of an approximation of a forbidden region is independent of particular data of a location problem.

Therefore the bounds we derived in Section 3 might not always be tight.

If we additionally take into account information we have for a particular problem instance and incorporate this information in the approximation algorithm we may get better bounds. In the following we discuss as an example an improved approximation algorithm for $1 / P / \mathcal{R} / l_{2}^{2} / \sum$ location problems to get more insight on how the previous results can be used for concrete solution algorithms.

By using exclusion rules we reduce the necessary number of approximation steps. Furthermore the next edge to be dissected is chosen with respect to the current optimum. In the following we present an algorithm and its exclusion rules. We assume the unrestricted unique optimum $\mathcal{X}^{*}$ to be given.

\section{Algorithm 4.1 Solving $1 / P / \mathcal{R} / l_{2}^{2} / \Sigma$}

Input: existing facilities $E x_{m}$, weights $w_{m}$, convex forbidden region $\mathcal{R}$ and unrestricted optimum $\mathcal{X}^{*}$

Output: approximate solution $\mathcal{X}_{\underline{P}}^{*}$.

1. Choose an value for the precision $\varepsilon$ and get from Theorem 3.1 an upper bound for the number of needed iterations. Here we may stop if this bound is much to high.

2. The first outer approximation is the rectangle parallel to the axes. The first inner approximation is the connection of the tangent points of the rectangle to $\mathcal{R}$.

3. We project $\mathcal{X}^{*}$ on the new generated edges of $\bar{P}$ and $\underline{P}$. (This is in the first iteration equivalent to solve the problems $1 / P / \bar{P} / l_{2}^{2} / \sum$ and $\left.1 / P / \underline{P} / l_{2}^{2} / \sum\right)$.

4. If the difference with respect to the objective function between the best projection on $\bar{P}$ and the best projection on $\underline{P}$ is less than $\varepsilon \rightarrow$ GOTO Step 7.

5. Delete all edges of $\bar{P}$, where

a) the objective value at the projection point is bigger than any objective value on $\underline{P}$, or where

b) the projection line intersects the boundary of $\mathcal{R}$ before hitting $\bar{P}$.

6. Take an edge of $\bar{P}$ with the smallest projection value, delete this edge from the list, execute the dissection with respect to this edge and GOTO Step 3. 


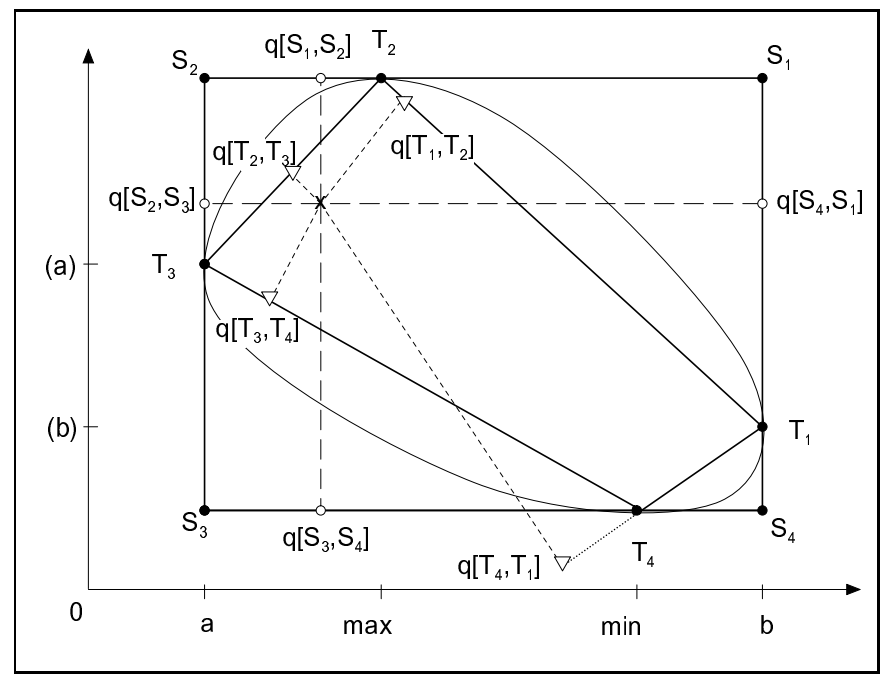

Figure 4: starting situation for a forbidden region $C$

7. Output: All best projections on the actual $\underline{P}$.

As one can easily see the efficiency of the exclusion rules depends on the location of $\mathcal{X}^{*}$ in the forbidden region. The closer $\mathcal{X}^{*}$ is to the boundary, the more obvious is the location of the restricted optimum and we can exclude intervals.

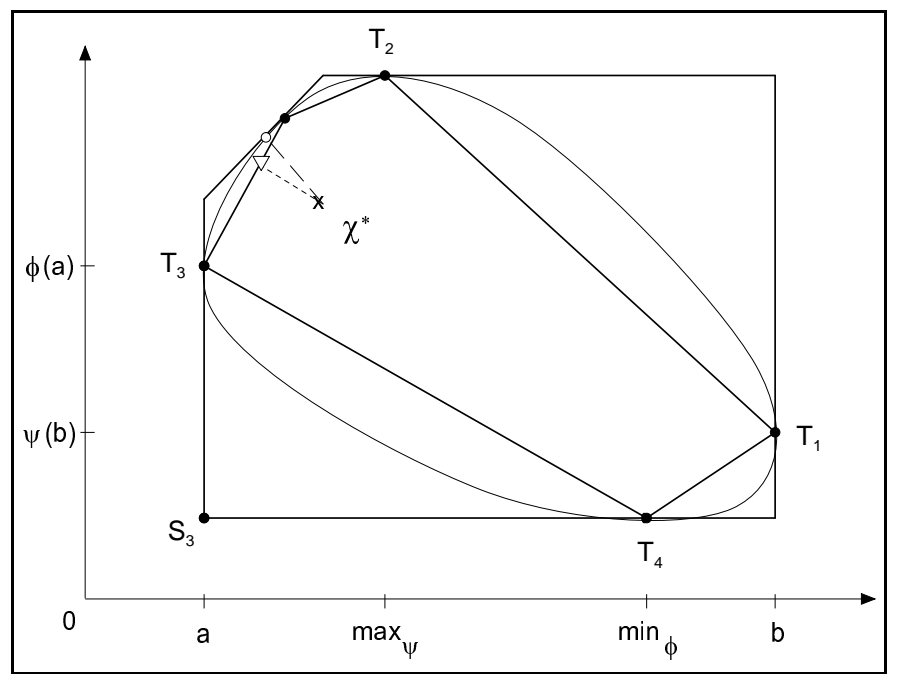

Figure 5: approximation polygons after one approximation step

In Figure 4 for edges $\left[T_{1}, T_{2}\right]$ and $\left[T_{4}, T_{1}\right]$ we observe two cases where the exclusion rules can be applied. The choice of edge $\left[T_{2}, T_{3}\right]$ to be the interval partitioned next is obvious. See Figure 5 for the illustration of such a partitioning. 
With a $\mathrm{C}++$ implementation of this algorithm on an RS6000 workstation we tested circles and ellipses as forbidden regions and generated location data by selecting points randomly in the plane.

For checking the error we took a circle as forbidden region since we are able to calculate the exact optimum in this case.

For our test we did 1000 runs, where in every run we generated 10 locations uniformly distributed in the plane with exponentially distributed weights. For the described forbidden regions the implementation delivered the following average results.

\begin{tabular}{|l|l|l|l|}
\hline$\varepsilon$-precision & $\begin{array}{l}\text { average } \\
\text { error }\end{array}$ & $\begin{array}{l}\text { average re- } \\
\text { lative error } \\
\text { with respect } \\
\text { to the real } \\
\text { optimum }\end{array}$ & $\begin{array}{l}\text { average } \\
\text { number of } \\
\text { iterations }\end{array}$ \\
\hline \hline $1 \mathrm{e}-01$ & 0.00833093 & 0.000200003 & 4.19923 \\
\hline $1 \mathrm{e}-02$ & 0.000802458 & $1.84018 \mathrm{e}-05$ & 6.52572 \\
\hline $1 \mathrm{e}-03$ & $6.57987 \mathrm{e}-05$ & $1.50866 \mathrm{e}-06$ & 9.35724 \\
\hline $1 \mathrm{e}-04$ & $7.60535 \mathrm{e}-06$ & $1.80115 \mathrm{e}-07$ & 11.6279 \\
\hline $1 \mathrm{e}-05$ & $7.6569 \mathrm{e}-07$ & $1.72459 \mathrm{e}-08$ & 14.2415 \\
\hline $1 \mathrm{e}-06$ & $6.1844 \mathrm{e}-08$ & $1.45573 \mathrm{e}-09$ & 16.7942 \\
\hline $1 \mathrm{e}-07$ & $7.13416 \mathrm{e}-09$ & $1.63229 \mathrm{e}-10$ & 19.234 \\
\hline $1 \mathrm{e}-08$ & $7.99617 \mathrm{e}-10$ & $1.87537 \mathrm{e}-11$ & 21.4575 \\
\hline $1 \mathrm{e}-09$ & $7.06953 \mathrm{e}-11$ & $1.67641 \mathrm{e}-12$ & 23.6035 \\
\hline $1 \mathrm{e}-10$ & $8.02235 \mathrm{e}-12$ & $1.86363 \mathrm{e}-13$ & 25.4161 \\
\hline
\end{tabular}

Table 1: Forbidden region is a circle with radius 4.

An average problem for $\varepsilon$-value of $1 \mathrm{e}-05$ needed 0.029 seconds of user time for the problems discussed in Table 1.

An average problem for $\varepsilon$-value of $1 \mathrm{e}-05$ needed 0.023 seconds of user time for the problems discussed in Table 2.

It should be noted that since the developed error bounds are worst-case bounds it is clear that the average performance is much better. In addition, also made use of exclusion rules. 


\begin{tabular}{|l|l|l|}
\hline$\varepsilon$-precision & $\begin{array}{l}\text { average } \\
\text { error }\end{array}$ & $\begin{array}{l}\text { average } \\
\text { number of } \\
\text { iterations }\end{array}$ \\
\hline \hline $1 \mathrm{e}-01$ & 0.0500437 & 2.83121 \\
\hline $1 \mathrm{e}-02$ & 0.00547363 & 4.94994 \\
\hline $1 \mathrm{e}-03$ & 0.000547272 & 7.01163 \\
\hline $1 \mathrm{e}-04$ & $5.21326 \mathrm{e}-05$ & 9.22261 \\
\hline $1 \mathrm{e}-05$ & $5.63873 \mathrm{e}-06$ & 11.0504 \\
\hline $1 \mathrm{e}-06$ & $5.45206 \mathrm{e}-07$ & 13.1568 \\
\hline $1 \mathrm{e}-07$ & $5.17894 \mathrm{e}-08$ & 15.6852 \\
\hline $1 \mathrm{e}-08$ & $5.63141 \mathrm{e}-09$ & 17.3576 \\
\hline $1 \mathrm{e}-09$ & $5.46529 \mathrm{e}-10$ & 19.6127 \\
\hline $1 \mathrm{e}-10$ & $5.06091 \mathrm{e}-11$ & 21.4583 \\
\hline
\end{tabular}

Table 2: Forbidden region is an ellipsoid of the form $a\left(x_{1}-m_{1}\right)^{2}+b\left(x_{2}-m_{2}\right)^{2}=r^{2}$, with $a=1, b=2$ and $r=2$. 


\section{Conclusions}

In this paper error bounds for the approximative solution of restricted planar location problems have been developed.

Using the sandwich algorithm to approximate forbidden regions, the number of approximation steps required to achieve a specified error bound for the solution is analysed. A great advantage of the methods used, is, that the number of approximation steps required to reach a certain quality can be given in advance. Computational results showed the practical efficiency of the approximation method.

There are further topics under research resulting from this general solution approach. A first interest is the consideration of mixed gauges. Obviously we arrive at very similar types of error bounds for different block norms. Objective functions that consist of combinations of block norms therefore should be easy to handle in terms of error bounds.

A second very fundamental research project is based on the polyhedral norm approach for

round norms. The property of polyhedral norms to be dense in the set of all norms leads to the attempt of solving location problems with general norms by problems using polyhedral norms. 


\section{References}

[AM94] Y.P. Aneja and Parlar M. Algorithms for weber facility location in the presence of forbidden regions and/or barriers to travel. Transportation Science, 28:70$76,1994$.

[BHR91] E.R. Burkard, H.W. Hamacher, and G. Rote. Sandwich approximation of unvariate convex functions with an application to separable convex programming. Naval Research Logistics, 38:911-924, 1991.

[DD84] W. Domschke and A. Drexl. An international bibliography on location and layout planning. Springer Verlag, Heidelberg, 1984.

[DM85] R. Durier and C. Michelot. Geometrical properties of the fermat-weber problem. European Journal of Operational Research, 20:332-343, 1985.

[Dre95] Zvi Drezner. Facility Location. A Survey of Applications and Methods. Springer Verlag, New York, 1995.

[DW91] Z. Drezner and G.O. Wesolowsky. The weber problem on the plane with some negative weights. INFOR, 29(2):87-99, 1991.

[FLMW92] R.L. Francis, F. Leon, J. McGinnis, and J.A. White. Facility layout and location: An analytical approach. Prentice-Hall, New York, 1992. 2nd Edition.

[Ham95] H.W. Hamacher. Mathematische Verfahren der Planaren Standortplanung. Vieweg Verlag, Braunschweig, 1995. 171 pages.

[HN94] H.W. Hamacher and S. Nickel. Combinatorial algorithms for some 1-facility median problems in the plane. European Journal of Operational Research, 79:340-351, 1994.

[HN95] H.W. Hamacher and S. Nickel. Restricted planar location problems and applications. Naval Research Logistics, 42:967-992, 1995.

[HN96] H.W. Hamacher and S. Nickel. Multicriteria planar location problems. European Journal of Operational Research, 94:66-86, 1996.

[HNS96] H.W. Hamacher, S. Nickel, and A. Schneider. Classification of location problems. Technical report, Department of Mathematics, University of Kaiserslautern, 1996. Submitted to Location Science.

[K9̈6] B. Käfer. Restriktive standortprobleme - ein approximationsansatz. Master's thesis, Fachbereich Mathematik, Universit t Kaiserslautern, 1996.

[Mic87] C. Michelot. Localisation in multifacility location theory. European Journal of Operationl Research, 31:177-184, 1987. 
[ND96] S. Nickel and E.-M. Dudenhöffer. Weber's problem with attraction and repulsion under polyhedral gauges. Technical report, University of Kaiserslautern, Department of Mathematics, 1996. Report in Wirtschaftsmathematik No. 5, submitted to Journal of Global Optimization.

[Nic95] S. Nickel. Discretization of Planar Location Problems. Shaker Verlag, Aachen, 1995.

[Rot92] G. Rote. The convergence rate of the sandwich algorithm for approximating convex functions. Computing, 48:337-361, 1992.

[Val64] F.A. Valentine. Convex Sets. Mc Graw-Hill, New York, 1964.

[WW85] J.E. Ward and R.E. Wendell. Using block norms for location modeling. Operations Research, 33:1074-1090, 1985. 